\title{
CHRONIC OTITIS MEDIA
}

\author{
F. McGuckin, M.D., F.R.C.S. \\ Department of Otolaryngology, Royal Victoria Infirmary, Newcastle upon Tyne
}

Chronic otitis media, because of its many facets, continues to be an absorbing problem. Each case presents a fresh challenge and there is no routine treatment. A brief review of the changes in our thinking over the past 30 years may be instructive, always bearing in mind that latter-day developments have not been so consistently successful that we can afford to belittle the achievements of our predecessors, however insecure may have been the foundations they laid.

\section{Description of the Disease}

A strict definition of the disease is virtually impossible and even mere description difficult; hence it is not surprising that in the literature the one is avoided and the other evaded. There is, however, some value in an attempt at description of certain aspects. For example, lesions and their sequelae may be grouped clinically in this fashion:

(1) Post-inflammatory conditions, showing the inactive residue of past damage in or adjacent to the middle-ear cleft.

(2) Recurrent otitis media, comprising those cases in which exacerbations are spaced by periods of genuine inactivity. This is not true chronic middle-ear disease, but it needs consideration because efficient management of acute episodes may limit potentially prolonged exacerbations.

(3) Continuous chronic otitis media, a blunderbuss term, but one useful enough to indicate a process, recognized or unrecognized, in which activity has been constant for two, or ten or 40 years, a process showing no easy tendency to self-limitation. Clinically, it is a condition sharply defined from the recurrent group wherein there is a repeated tendency to spontaneous improvement.

Such a scheme of description, with all its imperfections, is better than none, and there are many other methods of approach. The grouping given above is of more than theoretical use. It can be helpful in the clinical assessment of the individual problem, and it has proved useful in the application of the reconstructive techniques of tympanoplastic surgery.

If I make a special plea for some kind of description, it is because there have been too many claims about the results of treatment of materia $\vec{P}$ which may not be described at all, and therefore $\vec{\omega}$ we cannot know whether that material waso favourable or forlorn beforehand. Most observerso writing of otosclerosis have at least tried to be clinically exact, and of late to be pathologicallyw exact; there have been too few such efforts in the $e_{\triangle}$ wider and more difficult field of chronic otitis $\vec{\perp}$ media.

\section{Clinical Approach}

Over the years we have become increasinglyaware of the importance of the whole patient, of the need to relate otitic pathology to the total probleme. presented by a person. For example, if the $\vec{\circ}$ cardinal complaint is ever to be uncovered, the patient must be allowed to develop his histo He should be enabled to reveal why he comes ato this particular moment, after five or 15 years of continuous or recurrent ear trouble. The im-o portance of the cardinal complaint is nowadayso obvious. It may relate to deafness, discharge,, or discomfort, and if it is discomfort we must dis- $\overrightarrow{\overline{0}}$ cover whether it is mental unease, physical distress 3 or both. Suppose that the chief complaint is discharge, its quantity or its smell, then it is necessary to assess the chance of relief; if it iso deafness, one must consider the possibilities of restoration of function, and not by default leave the patient with the notion that somehow or other hearing will return; if discomfort or pain is the problem, one must decide whether reassurance, or minor therapy, or extensive surgery, is necessarys to future well-being. Where immediate and serious decisions are indicated, the surgeon is $=$ justified in imposing his will. In all other cir N cumstances, the prospects should be discussed with the patient and when surgical intervention is desirable he should understand that the primaryc aim is safety, or the production of a dry ear, or repair of function-and what may be the righte advice at the age of 35 may be the wrong advice at the age of 60 .

\section{Etiology}

Until recent times most text-book authors hel 
that chronic otitis media arose from unresolved or mistreated acute otitis media. J. S. Fraser ${ }^{1}$ was but one of many who accepted this view and, writing in the I920s he very rightly discussed the prolonged otorrhoeas which at that time resulted from the rapidly necrotizing complications of acute fevers. The etiology of an always undefined and sometimes ill-described disease was widely agreed, although as far back as 1874 Von Troltsch ${ }^{3}$ had allowed that some cases might arise insidiously, and that rarely the tympanum might be invaded from without-an opinion given some support by Albert Gray ${ }^{2}$ in 1910. If this general view of causation were correct, then the chronic ear problem should have been virtually abolished in the I940s, by which time efficient use of antibiotic remedies (in the existing biological balance) had made it clear that most acute lesions could be terminated satisfactorily by medical measures, provided the standards of physical and functional recovery were strict enough. It is common knowledge that the chronic ear problem is still very much with us and that therefore the traditional ideas about etiology are at best inadequte. Plainly there are now (and there probably always have been) large numbers of cases in which the acute-chronic sequence is not valid.

The first need is to recognize that we see an increasing proportion of cases in which disease is already chronic when the patient first has a symptom of any kind. Here is a clinical observation which for the moment means no more than it states, and it does not as yet necessarily imply something chronic from the beginning. It does mean that we have an obviously well-established desease not preceded by an overt acute episode, and it applies to children and adults alike. It is true that an apparently new lesion is often clearly a reactivation at the site of previous disease perhaps long quiescent or healed. Doubtless some of these old changes have followed on evident acute inflammations, but nowadays there is ample evidence to indicate that others have been quite symptomless at the time of the initial damage. In any event it is plainly necessary in current practice to jettison much of the traditional teaching about etiology since it is no longer tenable. (The interpretation of this statement requires judgment because the old teaching may still apply in great measure to certain parts of the world; for example conditions in Western Europe do not coincide with those in Alaska or in some areas of Africa.) However, there are reasons for supposing that we must revert to the suggestions so tentatively put forward by Von Troltsch and Albert Gray, and consider how far the notion of invasion from without the middle-ear cleft is valid.

A single clinical illustration, one of many, may help to justify the plea for a change in thinking. Some years ago a patient told me a dramatic story, roughly in the following words: " Two and a half weeks ago I suddenly went deaf in my left ear and lost the taste in the left side of my tongue.' At operation the attic showed a cholesteatoma which had invaded and disrupted the malleoincudal articulation and presumably caused pressure on the chorda tympani. Here was a chronic disease with sudden onset of two particular symptoms precisely described by an intelligent woman.

In addition, two hypotheses, one now generally accepted, the other as yet widely resisted, may give support to the argument. Negative-pressure conditions in the middle-ear cleft may arise from causes which are essentially non-inflammatory, varying from primary carcinoma in the orifice of the lower end of the Eustachian tube at one extreme, to a broad hyperplastic mass of adenoids at the other, or even from barotrauma to allergic oedema. (I do not intend to be bogged down here by disputation as to the presence or absence of free fluid as a necessary factor in deafness, because it is clear to me that deafness may be present with no more than change in pressure, positive or negative.) We know that the mesotympanum may revert to normal equilibrium, whilst some of the attic spaces may remain in a state of negative-pressure, because of their anatomical complexity and the variable ease of communication between one space and another. Supposing that all else has recovered we may be left with an indriven or obliterated Prussak's space. In this event, there is a small pouch, or potential pouch, consisting of three elements, an external keratinising cuticle, an internal mucosal sheet, and in between a very tenuous layer of connective tissue, representing locally the stiff mesoblastic tissue found elsewhere in the tympanic membrane. The small pouch is situated within the confines of the normal anatomical boundaries of the middle-ear cleft but as yet it lies outside the mucosal cleft proper. Careful scrutiny has shown that accumulation of sheets of pure keratin in the recess can produce dissolution of the barrier between the outside world and the tympanic cleft. Ruedi, among others, has demonstrated microscopically varying stages of the invasive process. Improved visual techniques have enabled us to arrest this kind of process whilst still relatively harmless, and so we have been provided with one means of preventing destructive cholesteatomatous disease in the epitympanum. Fairly wide contact with surgeons devoted to chronic ear work is my justification for saying that the hypothesis of invasion of keratin through the mid-attic is now accepted as a fact.

Now I wish to turn to the second hypothesis already described as widely resisted. The prob- 
lem of simple wax keratosis in the deep external canal, together with associated pathologies, is well-enough known, but it is frequently dismissed as a minor and easily corrected aural defect found on routine examination in such and such a percentage of all children presenting here or there. Yet if one adopts the precautionary idea that once there is a keratosis there may always be a keratosis, it will be seen that some of these simple lesions may reproduce themselves over a long period without giving rise to symptoms. I am suggesting that erosive meatal keratosis, sometimes called cholesteatoma of the outer ear, may insidiously produce a picture which eventually presents as something very like destructive chronic otitis media. Given proper vigilance and awareness of the possibilities, the real process may be detected and on occasion controlled by careful microscopic suction-clearance before it has reached the annulus or even at the stage when it has destroyed the middle layer of the drum-head but not yet entered the mucosal layer-in short before it has invaded the middle-ear cleft proper. It is worth considering whether the cause of posterior marginal perforation may not be keratotic invasion from without rather than pathology from within. The significance of the hypothesis, which for me is not completely proved at this date, lies in the sphere of preventive medicine. If there be anything in this notion, then preventive measures must begin with routine observation of the very young, with observation of the infant. If we can eliminate no more than a further Io per cent. of chronic ears by such vigilance we shall have achieved something, but I suspect that the proportion is an underestimate. On September 25, 1959, I was presented with a child of 14 months, presumed to have a chronic otitis for eight weeks. Three days later I carried out a careful suctionclearance. I know that this tympanum is intact and with proper observation I hope to keep it so.

I suggest therefore that accumulation of clinical facts has demonstrated the need for new concepts. These concepts in turn must be subjected to test, and modified or abandoned as experience dictates. Nevertheless, one overiding consideration cannot be dismissed. The management of the acute ear problem is not static. The balance between disease and therapy is a variable thing so that we must be prepared for adjustments from time to time.

\section{Pathology}

In pathology, there has been some improvement in the precise use of terminology and also a shift of emphasis on the role of certain factors. A clearer concept of the disease as a whole has in consequence emerged. Pathology is no longer regarded as a mere collection of polypi, necrotic mucosa, chronic osteitis, cholesteatoma and so on, but as something which may be assessed as accessible or inaccessible, as reversible or irreversible, as something in one case chiefly kept going by infection or in another case by a nutritional defect.

Lesions having a blood supply adequate to the diseased area will probably heal spontaneously, or may be encouraged to do so with a little assistance. Lesions in which nutrition is poor in whole or in part are likely to persist as continuous chronic pathologies or to show a pattern alternating between unstable quiescence and overt activity. In all this, infection frequently plays no more than a secondary role. It is useful to realize that there are some similarities between the chronic otitic picture and lesions like unhealed burns, varicose ulcers, long-bone osteitis, and persistent thoracic empyemata. This kind of thinking enables us to learn why so much of our treatment is fundamentally unsatisfactory. Proper appreciation of the significance of blood-supply will explain why an acute exacerbation may be brought under control, and why the basic chronic lesion is not at the same time terminated. As soon as the fresh hyperaemia ceases to exist, blood-borne remedies become inadequate or useless. Moreover, the same appreciation will show why so many attempt? at reconstructive surgery must fail; for example if the greater part of the ' bed ' prepared for the new graft consists of chalk and bare bone there can be little hope of survival. In addition, the greater the area of the graft unsupported by vital tissue, or one may say the greater the extent of the suspension-bridge of graft, the smaller the chance of success.

Two examples may serve to show how nutrition may be damaged: (I) A brief necrotising disease may destroy vascular supply very quickly, leaving conditions destined to become chronic. (2) A shallow chronic osteitis in the deep posterior bony canal may creep far enough to damage the annular region and so impair the blood-supply of the adjacent membrane.

Of necessity I can only survey the pathological aspect in the broadest fashion; but there is one point which deserves more detailed discussion. Argument about the matrix of cholesteatoma continues unabated. Some surgeons favour the retention of matrix as useful epithelium destined to assist rapid healing; other surgeons, among whom I include myself, regard matrix as disease which must be subjected to the most meticulous removal. It may be useful to dwell on the subject for a moment, because so much depends on which we mean by matrix, on the quality of the disease which is under treatment, and also on whether a 
clean piece of matrix is really to become useful healing tissue, or whether it is to be extruded and replaced by more reliable tissue.

I suggest that the clinical notion of matrix should include free keratin and everything down to the basal germinal layer from which is generated material gradually changing into free keratin. What lies beyond this germinal layer (which is not invariably demonstrated in microscopic section but which is nevertheless present somewhere) may vary from normal mucosa through innocuous fibrous tissue reaction, through reactive tissue of doubtfully reversible pathology, to gelatinous reactive tissue covering necrotic bone, showing patches of multiple minute sequestration or even destruction of the labyrinthine capsule or worse. The most innocent-looking sheet of matrix may cover anything, or lie over anything which its destructive tendency may have uncovered. The factors in cholesteatoma and its matrix making for destruction of hard tissue like bone are illunderstood but there is enough evidence to indidicate that pressure is a thoroughly unsatisfactory explanation. It is true that the production of free sheets of keratin is accompanied by liberation of phosphatase, but there is much to be done before we can assess the importance of enzymal and other changes. It is said that there is no essential difference between the thin epithelial lining of a healed ' radical' cavity and the structure of this cholesteatoma matrix. There is an essential difference in behaviour, and in the quantity of free keratin, and there are possibly variations in the germinal, malpighian, and granular layers. Finally, the most skilful and scrupulous attempt to excise cholesteatoma and its matrix may fail if there is invasion of cells in the solid angle, the peritubal region, the hypotympanum and so on.

That there are differing approaches to, and opinions about, pathology must be obvious, but we are at least moving towards a better appreciation of the factors involved and their significance in respect of proper clinical management of the disease.

\section{Management of the Disease}

The text-book authorities of the past showed almost explicit agreement about etiology. Moreover something else was at least implicit in the texts, namely an agreement about treatment. Failing control by minor therapy, wet or dry according to the school, then some type of socalled radical, modified radical, or quasi-radical procedure, or some form of eponymous operation, based on no particular surgical principle, should provide a reliable tap for turning off the flow of chronic discharge. The belief was strongest in those who saw least of their material afterwards.
A few men like J. S. Fraser in the 1920s, and Julius Lempert in the next decade, were exceptionally careful of surgical detail and in their hands results were above average. The truth, however, is that for those willing to see, not many of the advanced cases (of which we saw vast numbers) were terminated or even improved by any kind of medicine or surgery. Among the few which seemed to be arrested, relapse was almost the rule. Consider also how many lesions were 'dried up ' by a variety of powders, and how often beneath the crust of debris, disease continued its destructive course. The situation had to be confronted and it was obvious that new ideas and techniques were necessary.

The word treatment is not used here for the reason that we must manage situations differing from innocent perforations and minute erosions at one extreme, to chronic pan-otitis and diffuse intra-cranial invasion at the other.

The great merit of Lempert's earlier work in the chronic ear field was that it demonstrated the inadequacy of purely drainage procedures (of operations which only uncovered something) in advanced disease. Nevertheless, progress was limited by complex terminology and it was a change in this respect and not in technique alone which allowed the new gospel to diffuse. When George Shambaugh coined the word enchondralization he was not describing a new procedure, but he did provide us with a neat term of considerable value. What Lempert was really practising in advanced chronic otitic cases was excision of disease, though he did not coin the useful phrase excisive surgery. The concept, however, was a real advance, particularly when coupled with the new ideas of reversible and irreversible pathology, of accessibility and inaccessibility. The notion of the radical operation could now be discarded, and it had long been desirable that it should be, since it had never been specifically related to disease, to function, or anything very definite. We could now employ excisive surgery in one part of the middle-ear cleft and yet retain freedom to discuss conservation of function as a separate issue. Hence the way was prepared for the easy acceptance of the tympanoplastic advances of the last few years.

Before 1950 some workers had shown that timeconsuming microscopic techniques were justified by results, and that surgical revision might be usefully undertaken in some cases already submitted to as many as seven or eight previous operations. Improved cavity work was also shown to justify attempts at closure of very longstanding post-aural fistulae, if plastic principles were followed exactly. Out of all this there has grown a new standard of primary clinical assess-

.


ment which for some surgeons nowadays includes microscopic investigation at the first consultation. Improved optical equipment has made the task very much easier.

Coincident with the development of new ideas of management, observations of another kind were made, more by accident than design. Men began to appreciate that several differing physiologies might be consistent with useful, as opposed to normal hearing. For example, it was noted that a one-ossicle system plus a good membranous cover over the round window might provide nearnormal hearing. Such a modified physiological system was later called by Zollner ' columellahearing.' It was also observed that a mere hypotympanic baffle over the round window, aerated from the Eustachian tube, was consistent with satisfactory hearing, if the oval window were exposed and mobile. This, after all, was not surprising since it resembled the physiology of Lempert's fenestration operation for otosclerotic fixation of the oval window-one mobile window outside the tympanum, and one mobile window inside. These observations were made in cases of old damage wherein spontaneous healing had occurred after varying degrees of destruction. Attempts to reproduce similar conditions surgically gave rise to what we now call tympanoplastic surgery, the principles of which were succinctly codified by Wullstein in the period 1953-1955.

The advent of the new reconstructive concepts gave rise to a wave of untempered enthusiasm since many people thought that the solution of the chronic ear problem lay in doing something rather vague and then adding some kind of skin-graft. However, established principles had not changed and it presently became clear that these techniques were to be regarded as valuable refinements, that inherently they did nothing to eliminate the problem of chronic ear disease.
Tympanoplastic methods are largely applicable to conditions in which reliable healing is to be expected because basic principles have been $\frac{3}{\Phi}$ obeyed, and they produce the best functional 0 results in material which is inactive, or only active in a limited way beforehand.

I would mention the problem of healing in theo mastoid segment, a difficulty still with us and one음 certainly not eliminated by the large sheets of whole-skin or part-skin originally employed in $₫$ tympanoplastic surgery. The problem has received considerable attention and several attemptss have been made to solve it-for example the limita-. tion of the size of the operative cavity, the use of $\vec{\sim}$ living pedicle skin-flaps, the employment of pedicle-flaps of temporalis muscle, and so on I would repeat that, broadly speaking, recon- 3 . structive surgery is in principle easy where disease

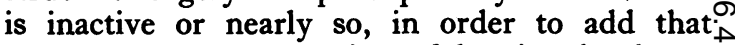
preservation or restoration of hearing is always $\vec{A}$ difficult and sometimes impossible in the presencen of advanced pathology.

We are not yet within sight of complete control 군 of chronic middle-ear disease, but we have for aconsiderable time been prepared to confess that the problem persists. The admission arises because men have been willing to acknowledge the bad, $\vec{\oplus}$ sometimes thoroughly bad results of methods? traditionally regarded as sound in principle and in practice. Too often there was no real princip? and too often has it been assumed that orthodox treatment must provide a good result.

\section{REFERENCES}

I. FRASER, J. S. (1927), 'Diseases of the Nose, Throat and Ear, ' ed. by A. Logan Turner, and edition, p. 321 .

2. GRAY, ALBERT (1910), 'The Ear and its Diseases,' p. 182, Bailliere, Tindall and Cox, London.

3. VON TROLTSCH (1874), 'The Surgical Diseases of the Ear, 3 p. 62 (translated by James Finton), The New Sydenhari Society, London.

References continu?d from page 235-The Management of Acute Renal Failure.

9. GAMBLE, J. L. (1947), Harvey Lectures, 42, 247.

ı. GIBSON, L. I. J. M. (1960), Lancet, i, 364 .

Ir. GROLLMAN, E. F., and GROLLMAN, A. (1959), f. clin. Invest., 38, 749 .

12. KOLFF, W. J. (1946), 'The Artificial Kidney,' Kok, Kampen, Holland.

13. Lancet, (1960), i, 371 (Editorial).

14. LOUGHRIDGE, L. W., MILNE, M. D., SHACKMAN, R., and WOOTTON, I. D. P. (1960), Lancet, i, 35 I.

15. LOWE, K. G. (1959), in Ross, E. J. (Ed.), 'Clinical Effects of Electrolyte Disturbances,' Pitman, London, 82.

16. MCCRACKEN, B. H., and PARSONS, F. M. (1958), Quart. F. Med., 27, 566 . ii, 885 .

18. McNAIR, T. J., and DUDLEY, H. A. F. (1959), Ibid., il, 365. N 19. MERONEY, W. H., and HERNDON, R. F. (1954), f. Amer. med. Ass., 155, 877.

20. PARSONS, F. M. (1959), Lancet, i, 148.

21. PIERCE, J. M. (1958), F. Urol., 80, 170.

22. SALISBURY, P. F. (1958), Arch. intern. Med., 101, 690

23. SCRIBNER, B. H. (1959), Northw. Med., Seattle, 58, 555.?

24. SHAW, G. (1959), Lancet, i, 15.

25. TAYLOR, W. H. (1957), Ibid., ii, 703.

26. TUCKER, D. A., WELLER, J. M., and MERRILL, J. P.T (1958), Amer. F. med. Sci., 236, 567. 